\title{
Evaluación del Bioinsumo de Fique Pulverizado (Furcraea spp) para el Control in vitro de Phytophthora infestans en papa (Solanum tuberosum $L$ )
}

\author{
Ruben D. Solarte, Oswaldo Osorio, Andres M. Hurtado, Diego F. Mejia \\ Universidad de Nariño, Facultad de Ingeniería Agroindustrial, Grupo de investigación Tecnologías \\ Emergentes en Agroindustria (TEA). Pasto-Colombia. (e-mail: osorio_oswaldo@udenar.edu.co)
}

Recibido Ago. 25, 2011; Aceptado Oct. 21, 2011; Versión final recibida Dic. 15, 2011

\begin{abstract}
Resumen
El objetivo de esta investigación fue evaluar el bioinsumo de fique pulverizado (Furcraea spp) para el control in vitro de Phytophthora infestans en papa (Solanum tuberosum L). Las condiciones óptimas de secado fueron: temperatura de entrada de $140^{\circ} \mathrm{C}$ y velocidad de $60 \mathrm{~m} / \mathrm{s}$. El producto obtenido se utilizó para enmendar un medio de agar tomate el cual se dispuso en cajas petri de 90 $\mathrm{mm}$ y se inoculó con discos de micelio del fitopatógeno de $1.2 \mathrm{~cm}$ de diámetro. El tratamiento óptimo inhibió en $100 \%$ el crecimiento del patógeno. Además se logró una reducción de la actividad acuosa del $37.4 \%$ lo cual permitió incrementar su preservación. Los resultados prueban que la técnica utilizada es altamente favorable para la conservación de las propiedades biológicas o biocidas del bioinsumo.
\end{abstract}

Palabras clave: Furcraea gigantea, Fique, Phytophthora infestans, secado por aspersión

\section{Evaluation of Antifungal activity of Spray-dried Fique (Furcraea spp) for in vitro control of Phytophthora infestans in potatoes (Solanum tuberosum $L$ )}

\begin{abstract}
The objective of this research was evaluating the antifungal activity of spray-dried Fique (Furcraea $s p p)$ for in vitro control of Phytophthora infestans in potatoes (Solanum tuberosum L). The optimal drying process variables studied were the entry temperature and air velocity of $140{ }^{\circ} \mathrm{C}$ and $60 \mathrm{~m} / \mathrm{s}$. The product obtained was amended in an agar medium tomato which was placed in petri containers, inoculated with $1.2 \mathrm{~cm}$ in diameter of pathogens discs. The optimum treatment inhibited $100 \%$ growth of the pathogen. Also, a reduction of water activity of $37.4 \%$ was achieved. This allowed increasing its preservation. The results showed that the technique is highly favorable for the conservation of bio-products of fungal properties.
\end{abstract}

Keywords: Furcraea gigantea, Fique plant, Phytophthora Infestans, spray drying 


\section{INTRODUCCIÓN}

El tizón tardío causado por Phytophthora infestans es una de las enfermedades más importantes en la papa y el tomate (Tian et al., 2000; Yang et al., 2003;. Bi et al., 2003). Este patógeno es especialmente problemático en las zonas andinas altas, donde dichos cultivos se desarrollan bajo condiciones de alta humedad y siembra continua durante todo el año (Mesa et al., 2008), lo cual favorece el desarrollo de la enfermedad, que es capaz de destruir en forma rápida la totalidad del follaje, (Latorre, 2004 y Agrios, 2005), razón por la cual se considera el problema más serio en la producción de este tubérculo a nivel mundial.

El control químico sigue siendo la principal medida para el control de las enfermedades, siendo los fungicidas metalaxil (metil $\mathrm{N}$-(metoxiacetil)- $\mathrm{N}$-(2,6-xili)-DL-alaninato), cimoxanil (1-(2-ciano-2metroximinoacetil)-3-etilurea) y dimetomorfo ((EZ)-4-[3-(4-chlorophenyl)-3-(3,4-dimethoxyphenyl) acryloyl]morpholine) los más ampliamente utilizados. El metil $\mathrm{N}$-(metoxiacetil)-N-(2,6-xilil)-DLalaninato es uno de los fungicidas fenilamidas, de gran actividad contra los oomicetos (Gui-ning ZHU, 2008), la utilización de este fungicida sistémico ha agravado la situación debido al uso intensivo, ya que esta práctica ha conllevado al desarrollo de poblaciones resistentes del patógeno (Alcalá et al., 1985; Rodríguez, 2001).

Jasso de Rodríguez (2011) evaluó la actividad inhibitoria in vitro de los extractos de Lippia graveolens Kunth, Agave lechuguilla Torr. Carnerosana, Yucca carnerosana (Trel.) Mc Kelvey y Yucca filifera Chaub., contra Rhizopus stolonifer, Colletotricum gloesporoides y Penicillium digitatum, obteniendo muy buenos resultados; con esto se concluye que los extractos naturales son una buena alternativa para el control de patógenos en productos agrícolas.

Preciado y Rangel (2006) y Rojas (2008) realizaron aproximaciones en la evaluación del potencial fúngico que tiene el jugo de fique en el control de Phytophthora infestans, encontrando que esta especie del genero Furcraea spp., utilizada principalmente en la extracción de fibras naturales, presenta cualidades fungicidas que pueden ser utilizadas en el manejo de este problema fitosanitario, ya que posee propiedades tensoactivas, plaguicidas y presenta contenidos de esteroides naturales entre los que se han encontrado saponinas y fitoesteroles (Preciado y Rangel, 2006; Rojas, 2008; Gómez y Vanegas, 2001), componentes que pueden interactuar como limitantes en el crecimiento del patógeno.

De la hoja de fique (Furcraea spp), solo el 4\% del total corresponde a la fibra, el $96 \%$ son desechos de los cuales el $70 \%$ es jugo (MADR, 2006), estos desechos generados, especialmente los líquidos sin un manejo adecuado ocasionan problemas de contaminación hídrica afectando la ictiofauna de las quebradas y la disponibilidad de los recursos hídricos para consumo humano y animal (Mojica y Paredes, 2004).

Tras diversas evaluaciones del jugo de fique a nivel in vitro como potencial inhibidor del fitopatógeno Phytophthora infestans causante de la gota en la papa, se concluyó que a una concentración de $75.000 \mu \mathrm{g} \mathrm{mL}^{-1}$ presento una inhibición satisfactoria; (Álvarez y Delgado, 2010).

La concentración efectiva reportada por Álvarez y Delgado (2010) es muy alta para fines de aplicación en campo, situación que dificulta su uso y aumenta los costos de manejo, debido a la necesidad de refrigeración y los altos costos de transporte en estado líquido, lo que lleva a la búsqueda de mejores alternativas entre las cuales está la pulverización, que permite un mejor manejo y mayor conservación del producto (Jaffe y Guerra, 2001).

Según Voigt (1982) el método de secado por aspersión se basa en atomizar la solución que va a ser secada en forma de gotas muy finas, en el seno de una corriente de gas caliente que generalmente es aire. En el proceso se forman partículas de geometría esférica, con aspecto de esferillas huecas, cuyo diámetro puede estar entre los $20 \mu \mathrm{m}$ y $200 \mu \mathrm{m}$. Tienen aspecto de espuma desecada y presentan gran solubilidad (Lopéz, 2010). El secado por aspersión es un proceso muy utilizado en la transformación de alimentos líquidos en polvo por evaporación del solvente. Comparado con otros procesos de evaporación, el secado por aspersión tiene la gran 
ventaja que el producto puede ser secado sin mucha pérdida de volátiles o componentes termolábiles (Teixeira et al., 1995).

El objetivo de la investigación fue determinar las condiciones adecuadas de pulverización del jugo de fique, y posteriormente verificar si el proceso de secado por aspersión tenía algún efecto sobre la actividad anti fúngica del jugo e igualmente que tan letal a nivel in vitro podría ser sobre el hongo Phytophthora Infestans.

\section{MATERIALES Y METODOS}

\section{Diseño experimental}

Se evaluó dos variables: temperatura entre 140 y $160^{\circ} \mathrm{C}$ y velocidad de aire entre 60 y $80 \mathrm{~m} / \mathrm{s}$, para lo cual se utilizó un diseño experimental completamente al azar (DCA), en el cual se evaluaron nueve tratamientos, donde las variables principales a considerar fueron las anteriormente mencionadas. T1F1 $\left(140{ }^{\circ} \mathrm{C} \times 60 \mathrm{~m} / \mathrm{s}\right)$, T1F2 $\left(140{ }^{\circ} \mathrm{C} \times 70 \mathrm{~m} / \mathrm{s}\right), \mathrm{T} 1 \mathrm{~F} 3\left(140{ }^{\circ} \mathrm{C} \times 80\right.$ $\mathrm{m} / \mathrm{s}), \operatorname{T} 2 \mathrm{~F} 1\left(150{ }^{\circ} \mathrm{C} \times 60 \mathrm{~m} / \mathrm{s}\right), \mathrm{T} 2 \mathrm{~F} 2\left(150{ }^{\circ} \mathrm{C} \times 70 \mathrm{~m} / \mathrm{s}\right), \mathrm{T} 2 \mathrm{~F} 3\left(150{ }^{\circ} \mathrm{C} \times 80 \mathrm{~m} / \mathrm{s}\right), \mathrm{T} 3 \mathrm{~F} 1\left(160{ }^{\circ} \mathrm{C} \times\right.$ $60 \mathrm{~m} / \mathrm{s})$, T3F2 $\left(160{ }^{\circ} \mathrm{C} \times 70 \mathrm{~m} / \mathrm{s}\right)$, T3F3 $\left(160^{\circ} \mathrm{C} \times 80 \mathrm{~m} / \mathrm{s}\right)$. Como variable respuesta se analizó el porcentaje de la actividad de producto pulverizado y él \% de crecimiento medio del micelio Phytophthora infestans.

\section{Obtención del bioinsumo de fique}

Se utilizaron hojas de fique de la variedad negra común (Furcraea gigantea Vent.), recolectadas en el municipio de Tambó Nariño y se llevaron a las instalaciones de la Planta Piloto de la Universidad de Nariño; allí se sometieron a limpieza y desinfección con el fin de eliminar todos los residuos contaminantes presentes en ellas. Posteriormente se realizó la extracción en un molino de rodillos diseñado para esta investigación; el jugo se filtró en lienzo y se fermentó por cuatro días a una temperatura de $35^{\circ} \mathrm{C}$ (Álvarez y Delgado, 2010). El bioinsumo de fique obtenido es tamizado a través de tamices 50 y 80 de la serie ASTM E- 11.

\section{Concentración}

La muestra se concentró con ayuda de un rota evaporador (Eyela OSB 2000), a $50^{\circ} \mathrm{C}$ y 10 psi por $1 \mathrm{~h}$ o hasta que alcance los $10^{\circ} \mathrm{Bx}$.

\section{Pulverización}

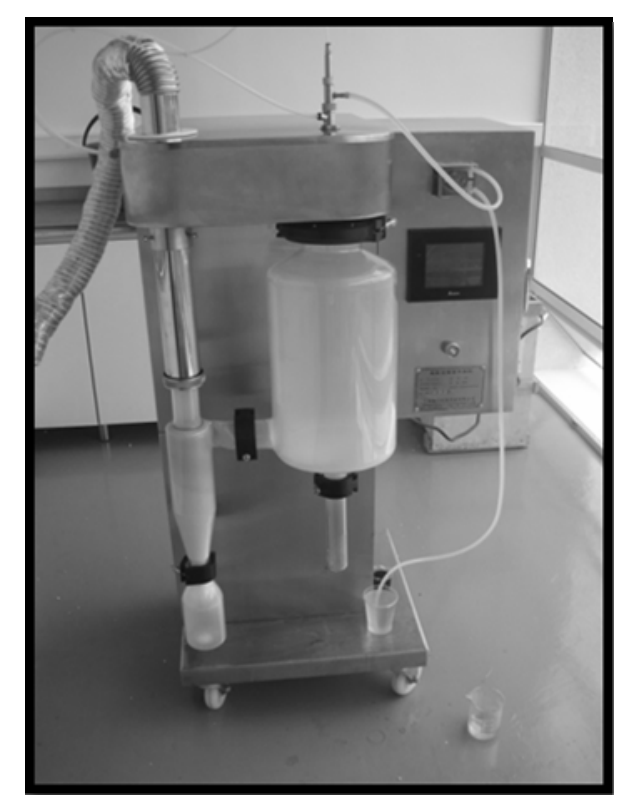

Fig. 1: Equipo de secado Spray 
El proceso de pulverización se llevó a cabo en un equipo de secado Spray a escala piloto, (referencia YC - 015) con una capacidad de $1500 \mathrm{ml} / \mathrm{h}$. Este se programó con cada uno de los parámetros de secado para un funcionamiento automático durante todo el proceso hasta su finalización.

\section{Medición de Actividad de agua}

La prueba se realizó en un equipo AquaLab LITE (7614AQUALITE) con un rango de medida de 0,03 - 1. Para la realización de la prueba se tomó una muestra aproximada de 0,3 gramos en el equipo y se procedió a realizar la lectura después de 3 min.

\section{Solubilidad}

Se tomó $1 \mathrm{~g}$ de muestra (base seca) la cual se colocó en $100 \mathrm{ml}$ de agua destilada a $30^{\circ} \mathrm{C}$ en un beaker con agitación continua, la suspensión se agitó por 5 min y luego se centrifugó a $3000 \mathrm{rpm}$ por $5 \mathrm{~min}$. Una alícuota de $25 \mathrm{ml}$ del líquido sobrenadante se transfirió a una caja petri, se pesó y se llevó a una estufa de secado a $105^{\circ} \mathrm{C}$ por $5 \mathrm{~h}$. Los sólidos recuperados se pesaron después del secado y se calculó el porcentaje de solubilidad por diferencia de pesos (Cano-Chauca et al., 2005; Shittu y Lawal, 2007).

\section{Evaluación In vitro del Bioinsumo pulverizado de fique}

Se evaluó la actividad inhibitoria in vitro de cada uno de los tratamientos a concentraciones de 10000 ug mL-1 por triplicado utilizando como testigo cajas petri sin bioinsumo, para un total de 33 unidades experimentales.

\section{Sensibilidad del patógeno Phytophthora infestans}

El comportamiento in vitro del patógeno se determinó por medio del crecimiento radial medio de Phytophthora Infestans, para esto se utilizó discos de micelio de $1,2 \mathrm{~cm}$ de diámetro del patógeno establecidos en cajas Petri con medio agar tomate enmendado con los diferentes tratamientos. El área de crecimiento se evaluó utilizando el programa gráfico ImageJ (Rasband, 2004) después de un período de incubación de 8 días a temperatura promedio de $18^{\circ} \mathrm{C}$. Se determinó el porcentaje de crecimiento utilizando la relación propuesta por Riveros et al., (2003):

$P C=\frac{D M C M-1,2 \mathrm{~cm}}{D M C A} \times 100$

Donde: $\mathrm{PC}=$ porcentaje de crecimiento; $\mathrm{DMCM}=$ diámetro medio de colonia creciendo en el tratamiento; $1,2 \mathrm{~cm}$ = diámetro del cilindro con micelio; y DMCA = diámetro medio de colonia sin enmendar.

Con base en el porcentaje de crecimiento se determinó la sensibilidad del aislamiento evaluado mediante la escala de sensibilidad propuesta por Shattock (1988) en donde:

Sensibles (S): Menor del $10 \%$ del crecimiento del testigo Intermedio (I): Entre 10 y $60 \%$ del crecimiento del testigo Resistente (R): Mayor del $60 \%$ del crecimiento del testigo

Los datos obtenidos se analizaron mediante análisis de varianza y prueba de comparación de medias LSD de Fisher a un nivel de significancia del 0,05 mediante el programa estadístico (Statgraphics plus 5.1, 2002).

\section{RESULTADOS Y DISCUSIÓN}

El bioinsumo pulverizado presenta una excelente solubilidad en agua, solvente normalmente utilizado en agroquímicos comerciales con presentación en polvo, los resultados mostraron un 
porcentaje de solubilidad del $98,75 \%$. Su solubilidad es excelente pero su humectabilidad observada es baja, por lo cual se requiere una muy buena agitación para su dilución por un tiempo aproximado de 5 min.

Tabla 1. Actividad anti fúngica del bioinsumo de fique pulverizado sobre el patógeno Phytophthora Infestans

\begin{tabular}{ccccc}
\hline Tratamiento & $\begin{array}{c}\text { Temperatura } \\
\left({ }^{\circ} \mathrm{C}\right)\end{array}$ & $\begin{array}{c}\text { Velocidad } \\
\text { del aire } \\
(\mathrm{m} / \mathrm{s})\end{array}$ & $\begin{array}{c}\text { Crecimiento medio } \\
\text { micelial }(\mathrm{cm})\end{array}$ & $\begin{array}{c}\text { Porcentaje de } \\
\text { Crecimiento }(\%)\end{array}$ \\
\hline T0 & \multicolumn{2}{c}{ Testigo Absoluto } & 5,425 & 100,00 \\
TB & \multicolumn{2}{c}{ Bioinsumo Líquido } & 2,345 & 21,11 \\
T1F1 & 140 & 60 & 1,201 & 0,00 \\
T1F2 & 140 & 70 & 1,296 & 1,77 \\
T1F3 & 140 & 80 & 1,354 & 2,84 \\
T2F1 & 150 & 60 & 1,417 & 3,98 \\
T2F2 & 150 & 70 & 1,435 & 4,29 \\
T2F3 & 150 & 80 & 1,455 & 4,72 \\
T3F1 & 160 & 60 & 1,489 & 5,36 \\
T3F2 & 160 & 70 & 1,536 & 6,19 \\
T3F3 & 160 & 80 & 1,566 & 6,76 \\
\hline
\end{tabular}

En la evaluación del bioinsumo pulverizado a una concentración de $10.000 \mu \mathrm{g} \mathrm{mL}-1$ se determinó que todos los tratamientos tienen efecto inhibitorio sobre el crecimiento de $P$. Infestans a nivel in vitro tal como se muestra en la tabla 1; esto se aprecia mejor en la figura 2, frente al testigo T0 y TB. Según la escala de Shattock todos los tratamientos (T1F1 a T3F3) presentan crecimientos por debajo del $10 \%$ indicando un nivel de sensibilidad; el testigo (TB) bioinsumo de fique líquido presento un nivel intermedio en la escala. El buen efecto del bioinsumo de fique pulverizado con respecto al liquido, puede deberse a la concentración de los metabolitos secundarios presentes en esta especie.

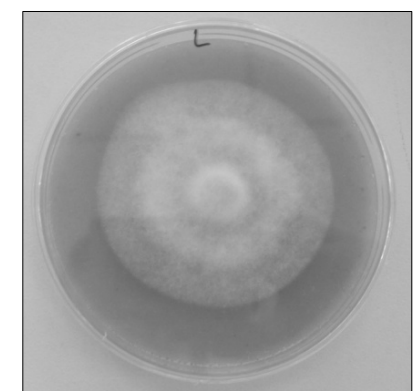

Testigo Absoluto T0

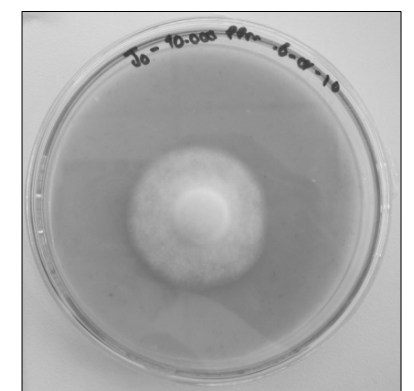

Testigo Bioinsumo liquido TB

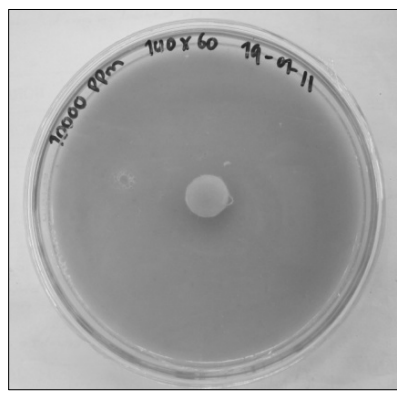

Bioinsumo pulverizado T1F1

Fig. 2: Sensibilidad del patógeno Phytophthora infestans al bioinsumo de fique pulverizado

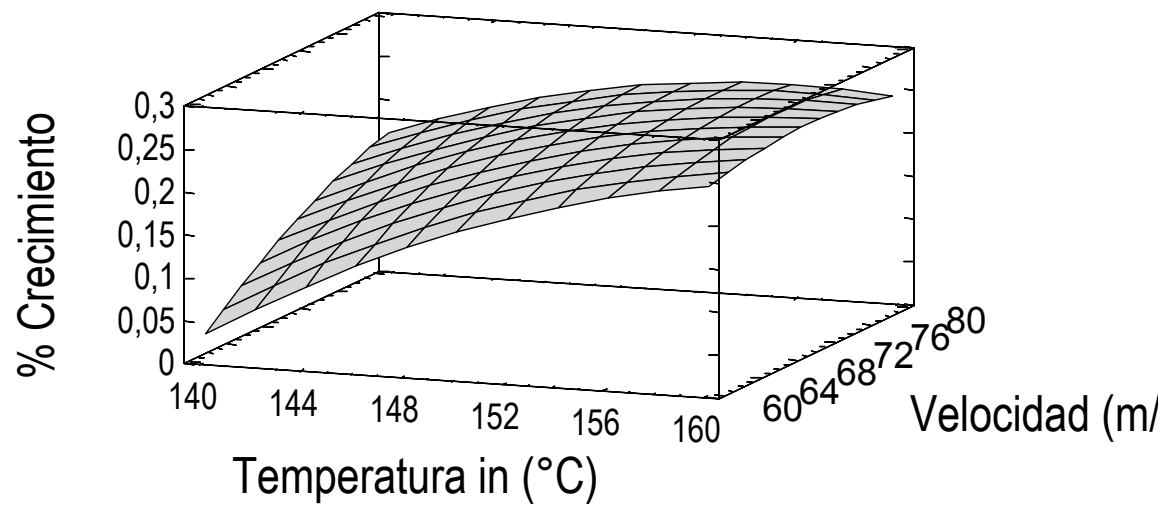

Fig. 3: Datos transformados: Arco Seno de raíz cuadrada de X/100 
Mediante el análisis del diseño experimental planteado se encontró que existe diferencia estadísticamente significativa entre los factores analizados con un $\mathrm{P}<0,05$ a un nivel de confianza del $95 \%$. El análisis mostro que el factor más influyente dentro del proceso de secado Spray es la temperatura, seguido de la velocidad del aire tal como se aprecia en la figura 3 . El modelo presenta un excelente ajuste, con una alta correlación ( $R 2=98,0478 \%$ ) y se minimizan las posibles causas de error a un $97,083 \%$.

La superficie de respuesta (Figura 3), muestra una superficie en ascenso, sin formas complejas, y sin curvaturas pronunciadas. Las condiciones óptimas de secado con base en el grado de inhibición del bioinsumo en polvo sobre el hongo Phytophthora infestans está representada por el punto mínimo en la superficie con $\mathrm{T}\left(140^{\circ} \mathrm{C}\right)$ y $\mathrm{F}(60 \mathrm{~m} / \mathrm{s})$.

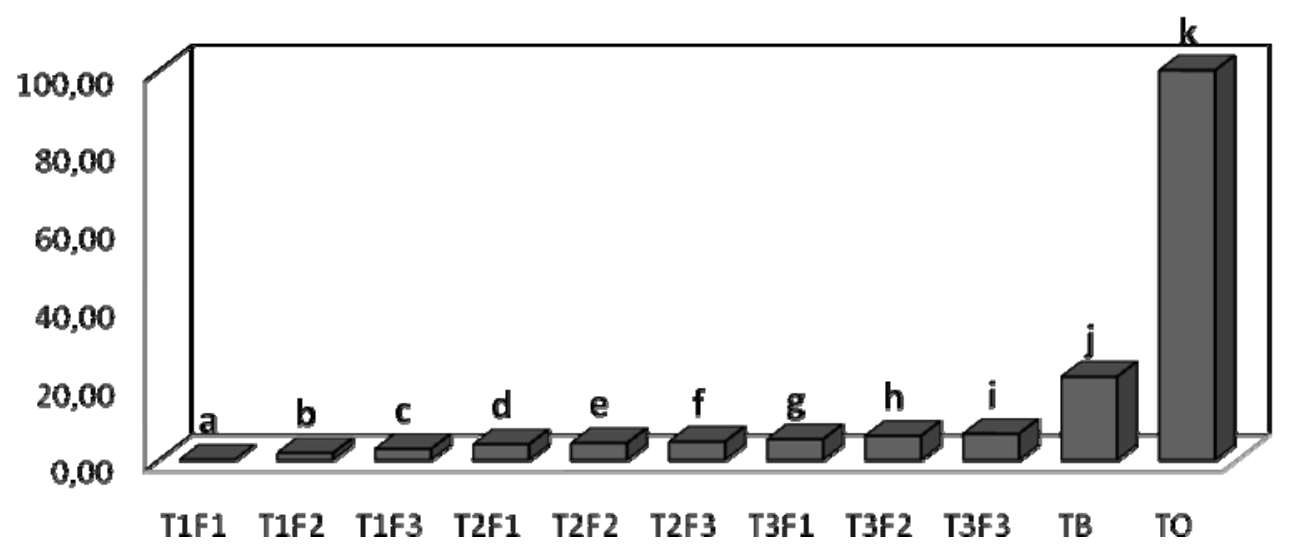

Fig. 4: Porcentaje de crecimiento entre los tratamientos evaluados y los testigos (Letras iguales no hay diferencia estadística)

Mediante comparación estadística entre cada uno de los tratamientos y los dos testigos evaluados se encontró diferencia estadísticamente significativa $(P<0,01)$ a un nivel de confianza del $99 \%$ (Fig. 4), mostrando que todos los tratamientos muestran un efecto significativo sobre la inhibición del fitopatogeno; por otro lado con el fin de determinar cuál de los tratamientos fue el mejor se realizo la prueba de Duncan, mediante la cual se obtiene que todos los pares de tratamiento presentan diferencias estadísticamente significativas, pero que la mejor media es la del tratamiento T1F1, que tal como lo muestra la figura 2 es el principal inhibidor del hongo Phytophthora infestans.

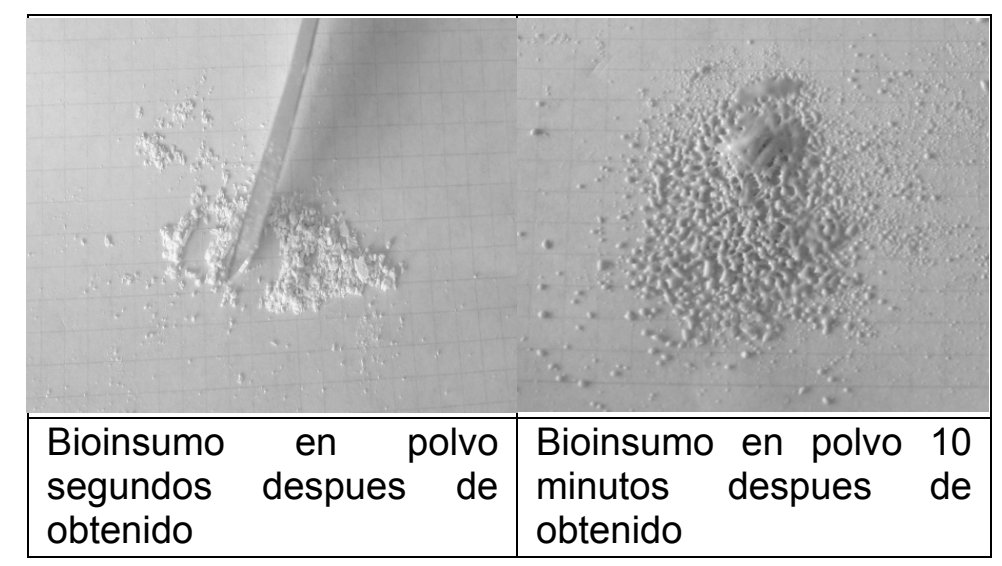

Fig. 5: Bioinsumo pulverizado sometido al ambiente.

La actividad del agua (Aw) tras el proceso de pulverización disminuyo de 0,975 a 0,365 presentando una reducción del 37,4 \%; sin embargo tras verse sometido al ambiente el bioinsumo pulverizado presentó propiedades higroscópicas (Fig. 5), las cuales llevaron a buscar las condiciones óptimas de conservación, para lo cual se evaluó dos tipos de empaque, bolsas 
plásticas resellables y tubos de vidrio ámbar con capacidad aproximada de 2 gramos. Los mejores resultados para la conservación se evidenciaron en los tubos de vidrio, conservando adecuadamente el bioinsumo. Para posteriores ensayos se recomienda evaluar un tipo de agente encapsulante con el fin de mejorar las condiciones de conservación.

\section{CONCLUSIONES}

La método pulverización empleado condujo a una reducción considerable en el porcentaje de crecimiento del patógeno Phytophthora infestans.

Igualmente esta técnica permitió la reducción en la actividad de agua, la cual está directamente relacionada con el grado de inhibición del bioinsumo sobre el fitopatogeno y a su vez con la conservación del producto.

De los tratamientos evaluados a nivel in vitro, el tratamiento T1F1 $\left(140^{\circ} \mathrm{C} \times 60 \mathrm{~m} / \mathrm{s}\right)$ aplicado a una dosis de $10.000 \mathrm{mg} \mathrm{mL}^{-1}$ obtuvo el mejor resultado en inhibición del hongo Phytophthora infestans con un porcentaje de crecimiento del $0 \%$.

Por otro lado el bioinsumo en polvo presenta una muy baja humectabilidad la cual contrasta con su alta solubilidad en agua, siendo esta del $98,75 \%$.

En vista de los resultados, el bioinsumo pulverizado obtenido a través de esta investigación tiene una gran ventaja en cuanto al manejo, transporte y conservación; por tanto su posible utilización en campo en un futuro brindaría excelentes ventajas competitivas frente agroquímicos convencionales e igualmente al bioinsumo de fique en estado liquido, situación que justifica una mayor investigación de este a nivel de campo.

\section{AGRADECIMIENTOS}

A la Vicerrectoria de Investigaciones de la Universidad de Nariño, Colombia, por la financiación del proyecto.

Al Grupo de Investigación Tecnologías Emergentes en Agroindustria TEA - Facultad de de Ingeniería Agroindustrial, Universidad de Nariño.

\section{REFERENCIAS}

Agrios, G. Plant pathology. 5 ed. México: Ed. Limusa, 826 - 827, (2004).

Alcalá, D; J, Marcano y A, Pire. Presencia de cepas del hongo Phytophthora infestans (Mont) de Bary resistentes a metalaxyl en siembras de papa del estado Lara. Agronomía Tropical, 35(1-3), 43-55 (1985)

Álvarez, D y Delgado, D. Sensibilidad in vitro de Phytophthora infestans al extracto de fique (furcraea gigantea vent) y fungicidas sistémicos. Tesis de Titulación, Programa de Ingenieria Agronomica, Universidad de Nariño, San Juan de Pasto, Colombia (2010).

Bi, C. W; Wang, Z; Che, X. Resistance of Phytophthora infestans to metalaxyl and the strategy for control. Southwest China Journal of Agricultural Sciences, 16, 68-70 (2003).

Cano-Chauca, M; y otros 3 autores. Effect of the carriers on the microstructure of mango powder obtained by spray drying and its functional characterization. Innovative Food Science \& Emerging Technologies, 6(4), 420-428 (2005). 
Gómez, M y Vanegas, E. Evaluación de la producción de esteroides a partir del jugo de fique con Cunninghamella spp, Tesis pregrado, Universidad Pontificia Bolivariana de Medellín, Facultad de Ingeniería Química, (2001).

Jaffe, W y Guerra, M., El Lactovisoy en el combate de la desnutrición infantil, An Venez Nutr, 14(2), 99-102 (2001).

Jasso de Rodriguez. D.; y otros 6 autores. In vitro antifungal activity of extracts of Mexican Chihuahuan Desert plants against postharvest fruit fungi, Industrial Crops and Products, 34 (1), 960-966 (2011).

Latorre, G.B., Enfermedades de las plantas cultivadas, $6^{\text {a }}$ Edición, 628, Ediciones Universidad Católica de Chile, Santiago, Chile (2004)

López, O., Microencapsulación de sustancias oleosas mediante secado por aspersión, Cubana Farm, 44 (3), 381-389 (2010).

Mesa, V.; y otros 6 autores. Variabilidad genética de aislamientos de Phytophthora infestans procedentes del suroeste de Colombia, Rev. Iberoamericana de Micologia, 25( 3), 167-172, (2008).

Ministerio de Agricultura y Desarrollo Rural (MADR)., Guia ambiental del subsector Fiquero. $2^{\text {da }}$ Edición, Bogota, 27-28 (2006).

Mojica, A y Paredes, J. El cultivo del fique en el departamento de Santander. Bucaramanga (Santander, Colombia), Centro Regional de Estudios Económicos. Bucaramanga, 154-155 (2004).

Osorio, L.; Valverde, F y Bonilla, C. Evaluation of cuban hemp, nut sedge, johnson grass and herb of grace extracts in weed control. Acta Agronómica, 58 (2), 103-107 (2009).

Palacios-Nava, M y Moreno-Tetlacuilo, L. Diferencias en la salud de jornaleras y jornaleros agrícolas migrantes en Sinaloa, México, Salud Pública Mex, 46 (4), 286-293 (2004).

Preciado, D y Rangel, E., Extracción de un biofungicida a partir del jugo de fique (Furcraea spp), Universidad pontificia bolivariana, Trabajo de grado Facultad de ingeniería química, pág. 78, (2006).

Rasband, W.S. Image J., National Institutes of Health, Bethesda, Maryland, USA, http://rsb.info.nih.gov/ij/. 1997-2004.

Rivas, Z.; y otros 5 autores. Contribución de principales ríos tributarios a la contaminación y eutrofización del lago de Maracaibo. Ciencia ,13 (1), 68-77 (2005).

Riveros, F.; y otros 4 autores. Reistencia de Phytophthora infestans (Montagne) de Bary a Metalaxyl en cultivo de papas en el norte de chile. Agricultura técnica, 63 (2), 117-124 (2003).

Rodríguez, D., Compatibilidad sexual, reacción a metalaxil y agresividad de aislamientos venezolanos de Phytophthora infestans, HYPERLINK "http://www.cdc.fonacit.gob.ve/cgiwin/be_alex.exe?Acceso=T052100011201/0\&Nombrebd=fonacit" Fitopatología Venezolana , 14 (1), 13-18 (2001). 
Rojas, M., Características fisicoquímicas del jugo de fique (Furcraea spp), elaboración y evaluación de un biofungicida útil en el control agroecológico de la gota (Phytophthora infestans) en la papa, Tesis de Titulación, Programa de Ingenieria Agroindustrial, Universidad de Nariño, San Juan de Pasto, Colombia (2008).

Salazar, C., Dinámica temporal de las poblaciones de Phytophthora infestans a los fungicidas Metalaxyl, Cymoxanil y Propamocarb en cultivos de papa, Tesis de magister, Facultad de Agronomía, Universidad Nacional de Colombia, Bogota-Colombia (2000).

Shattock, R., estudies on the inheritance o resistance to metalaxyl in Phytophthora infestans, Plant Pathology, 37(1), 4-11 (1988).

Shittu, T y Lawal, M. Factors affecting instant properties of powdered cocoa beverages. Food Chemistry, 100, 91-98 (2007).

Statgraphics plus 5.1. Statistical Graphics Corp (2002).

BIBLIOGRAPHY V 9226 Teixeira, P, y otros, Survival of Lactobacillus delbrueckii ssp. Bulgaricus following spray drying, Dairy Science, 78 (5), 1025-1031 (1995).

Tian M Y.; y otros 3 autores. Isolation and purification of Phytophthora infestans disease germ. Plant Protection, 26, 36 (2000).

Torres, D y Capote, T., Agroquímicos un problema ambiental global: uso del análisis químico como herramienta para el monitoreo ambiental. Ecosistemas, 13 (3), 2-6 (2004).

Voigt, R. Tratado de tecnología farmacéutica. Zaragoza: Editorial Acribia; $64-65$ (1982).

Yang Y H.; y otros 3 autores. The resistance of Phytophthora infestans isolates to metalaxyl. Acta Phytophylacica Sinica, 30, 57-62 (2003).

Yanza, E., Diseño de un secador por atomización a nivel piloto para jugo concentrado de tomate de árbol, Tesis Pregrado, Universidad Nacional de Colombia, Facultad de Ingeniería y Arquitectura, Manizales-Colombia, (2003).

ZHU, Gui-ning.; y otros seis autores, Sensitivities of Phytophthora infestans to Metalaxyl, Cymoxanil, and Dimethomorph, Agricultural Sciences in China, 7( 7), 831-840 (2008). 
